\title{
Safe electrode trajectory planning in SEEG via MIP-based vessel segmentation
}

\author{
Davide Scorza ${ }^{\mathrm{a}, \mathrm{c}, \mathrm{e}}$, Sara Mocciaa ${ }^{\mathrm{a}, \mathrm{b}}$, Giuseppe De Luca ${ }^{\mathrm{a}}$, Lisa Plaino ${ }^{\mathrm{a}}$, Francesco Cardinale ${ }^{\mathrm{d}}$, \\ Leonardo S. Mattos ${ }^{\mathrm{b}}$, Luis Kabongo ${ }^{\mathrm{c}}$, , and Elena De Momi ${ }^{\mathrm{a}}$ \\ ${ }^{a}$ DEIB, Politecnico di Milano, Milan, Italy \\ ${ }^{b}$ Department of Advanced Robotics, Istituto Italiano di Tecnologia, Genoa, Italy \\ ${ }^{c}$ e-Health and Biomedical Applications Department, Vicomtech-IK4, Donostia-San Sebastián, \\ Spain \\ ${ }^{d}$ Claudio Munari Centre for Epilepsy and Parkinson surgery, Niguarda Ca' Granda Hospital, \\ Milan, Italy \\ eBiodonostia Health Research Institute, Donostia / San Sebastián, Spain
}

\begin{abstract}
Stereo-ElectroEncephaloGraphy (SEEG) is a surgical procedure that allows brain exploration of patients affected by focal epilepsy by placing intra-cerebral multi-lead electrodes. The electrode trajectory planning is challenging and time consuming. Various constraints have to be taken into account simultaneously, such as absence of vessels at the electrode Entry Point (EP), where bleeding is more likely to occur. In this paper, we propose a novel framework to help clinicians in defining a safe trajectory and focus our attention on EP. For each electrode, a Maximum Intensity Projection (MIP) image was obtained from Computer Tomography Angiography (CTA) slices of the brain first centimeter measured along the electrode trajectory. A Gaussian Mixture Model (GMM), modified to include neighborhood prior through Markov Random Fields (GMM-MRF), is used to robustly segment vessels and deal with the noisy nature of MIP images. Results are compared with simple GMM and manual global Thresholding (Th) by computing sensitivity, specificity, accuracy and Dice similarity index against manual segmentation performed under the supervision of an expert surgeon. In this work we present a novel framework which can be easily integrated into manual and automatic planner to help surgeon during the planning phase. GMM-MRF qualitatively showed better performance over GMM in reproducing the connected nature of brain vessels also in presence of noise and image intensity drops typical of MIP images. With respect Th, it is a completely automatic method and it is not influenced by inter-subject variability.
\end{abstract}

Keywords: SEEG, vessel segmentation, trajectory planning, safety neurosurgery

\section{INTRODUCTION}

Focal epilepsy is a neurological disorder that causes uncontrolled electrical activity in localized brain areas. Focal epilepsy surgery requires prior identification of the Epileptogenic Zone (EZ), namely the area to be resected and/or disconnected. The EZ identification can be performed with Stereo-ElectroEncephaloGraphy (SEEG), a minimally invasive image-guided surgical procedure. During SEEG, surgeons implant intra-cerebral electrodes into patient brain to record brain activity and localize the EZ.

In the last years, technology improvements in the fields of imaging, robotics and image guided surgery have supported SEEG advancements, ${ }^{1}$ increasing both procedure accuracy and patient safety. ${ }^{2,3}$ In this scenario, research on computer-aided trajectory planning is spreading. The maximization of the distance from vessels is one of the most popular criteria adopted to define the best trajectory. ${ }^{4,5}$

Manual vessel segmentation is a tedious, time-consuming task, characterized by high inter-subject variability. ${ }^{6}$ Fraz et al. ${ }^{6}$ reviewed in 2012 different studies on automatic and semi-automatic vascular segmentation, classifying algorithms as (i) pattern recognition techniques, (ii) matched filtering, (iii) vessel tracking/tracing, (iv)

Davide Scorza: E-mail: dscorza@vicomtech.org, Telephone: +[34] 943309230 


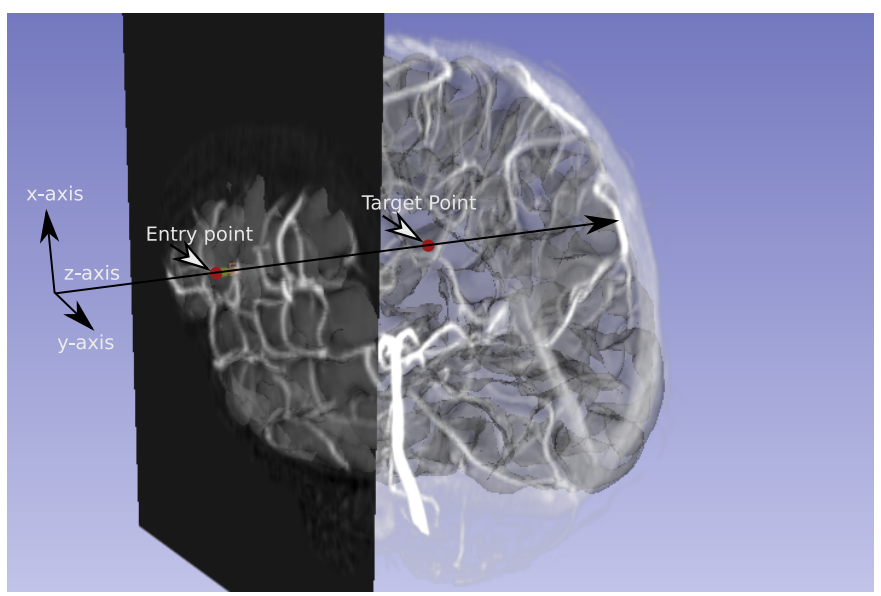

Figure 1. Maximum Intensity Projection (MIP) extraction. For each electrode, the MIP image is obtained projecting a portion of the volume on the perpendicular plane to the electrode insertion direction.

mathematical morphology, (v) multiscale approaches, (vi) model based approaches and (vii) parallel/hardware based approaches. Simple thresholding was used, ${ }^{4,7}$ with the main drawback of being sensitive to noise and image inhomogeneities. Du et al. ${ }^{8}$ used thresholding on a pre-processed image where vessels were enhanced with using the popular multiscale vesselness presented in. ${ }^{9}$ Gradient-driven level-set method was used to segment vessels in.$^{10}$ Level set was initialized with the multiscale vesselness. ${ }^{9}$ A multiscale approach based on tensor voting was also used. ${ }^{11}$

Despite the good results of the previous studies, further research is needed to increase the accuracy of vascular segmentation and, accordingly, the safety of electrode insertion. Particular attention should be given to the cortical electrode Entry Point (EP), which is the most risky and challenging point. ${ }^{3}$ This is due to the larger probability of causing bleeding while drilling skull or ablating dura mater.

In this work, we propose a method to assist the surgeon in the definition of the whole trajectory and, in particular, at the EP. Hence, w automatically segment brain vessels on Maximum Intensity Projection (MIP) images obtained from the first centimeter of brain, measured along the planned electrode direction. Vessels are here segmented with Gaussian Mixture Model (GMM), duly modified to include Markov Random Fields (GMM-MRF) modeling as to privilege connected set of pixels. This is particularly useful to compensate possible inaccurate vessel segmentation, due to noise or image inhomogeneities. Furthermore, the proposed method visually supports surgeons in understanding if the trajectory at the EP can be considered safe. To the best of authors' knowledge, this is the first attempt of including such clinically relevant constraint as support for the electrode trajectory planning.

This paper is organized as follow: Sec. 2 explains the methods and material used in this research. Sec. 3 deals with qualitative and numerical evaluation of the proposed approach. To conclude, Sec. 4 summarizes this research commenting the results and proposing future work.

\section{METHODS}

This section presents the details of the segmentation process: Sec. 2.1 describes MIP images generation, which provides high contrasted vessels. Sec. 2.2 presents and explains the vessel segmentation algorithm. Sec. 2.3 and Sec. 2.4 report on patient data and performance evaluation indexes, respectively.

\subsection{MIP Extraction}

A crucial requirement during SEEG electrode trajectory planning is vessel avoidance, especially at the EP, as described in Sec. 1. Accordingly, cerebral vessel enhancement in CT images through the injection of a contrast medium is a common practice in different epilepsy centers. ${ }^{12,13}$ 
In clinical practice, usually surgeons check interactively the whole trajectory looking for vessels presence, and modify it accordingly. Hence, we developed a python module in 3D Slicer, ${ }^{14}$ which allows surgeon to place EP and TP on vascular images (e.g. CT-DSA ${ }^{3}$ ) defining an electrode trajectory. An orthogonal Reference Frame $(\mathrm{RF})$ with origin in the EP and z-axis towards the Target Point (TP) is built, implementing the so-called Probe Eye View.

Nonetheless, vessel recognition is not an easy task and noisy spots could lead to misclassification. Therefore, in this paper we generate MIPs on Probe Eye View plane considering a user-defined portion of volume, enhancing vessel visualization and simplifying the understanding of their presence along the trajectory (Fig. 1). To further focus on the EP, MIP images are automatically obtained from the first centimeter and subsequently processed to segment vessels as explained in Sec. 2.2.

\subsection{Vessel Segmentation}

In this paper, vessel segmentation on MIP images is performed through GMM. GMM is a statistical modeling that aims to exploit the statistical differences in intensity distribution between vessels (bright) and background (dark). Therefore, it represents the most natural way to deal with the noisy nature of MIP images. ${ }^{15}$

One of the main drawback of a classical GMM-based segmentation is the assumption of pixel independence, which could represent a limit in presence of high noise level. This is particularly true when dealing with MIP images, where the projection of noise causes isolated high intensity pixels that may be misclassified as vessels. ${ }^{16}$ To solve this issue, MRFs can be included in the GMM-based segmentation formulation to incorporate spatialcontext information by relaxing the pixel independence assumption.

The GMM formulation considers image intensities $\mathbf{x}=\left(x_{1}, x_{2}, \ldots, x_{N}\right)$ as a realization of a causal variable: $\mathbf{X}=\left(X_{1}, X_{2}, \ldots, X_{N}\right)$, where the $X_{i}$ 's are independent and identically distributed with probability density functions $f\left(x_{i}\right)$. Considering $K$ classes, each one associated to a density function $f_{k}\left(x_{i} \mid \theta_{k}\right)$, the probability of a pixel to belong to the $k^{\text {th }}$ class can be approximated by a Gaussian function:

$$
f_{k}\left(x_{i} \mid \theta_{k}\right)=\frac{1}{\sqrt{2 \pi \sigma_{k}^{2}}} e^{\frac{\left(x_{i}-\mu_{k}\right)^{2}}{2 \sigma_{k}^{2}}}, \quad \theta_{k}=\left(\mu_{k}, \sigma_{k}^{2}\right)
$$

where $\theta_{k}$ is a vector of two parameters: mean $\mu_{k}$ and variance $\sigma_{k}^{2}$ of the $k^{\text {th }}$ Gaussian distribution. The parametrized probability density function can be written as follow:

$$
f\left(x_{i} \mid \Psi\right)=\sum_{k=1}^{K} \pi_{k} f_{k}\left(x_{i}, \theta_{k}\right)
$$

where $\pi_{k}$, called mixing proportions, satisfy:

$$
0 \leq \pi_{k} \leq 1 \quad \text { and } \quad \sum_{k=1}^{K} \pi_{k}=1
$$

and

$$
\boldsymbol{\Psi}=\left(\pi_{1}, \pi_{2}, \ldots, \pi_{k}, \Theta\right) \quad \text { with } \quad \boldsymbol{\Theta}=\left(\theta_{1}, \theta_{2}, \ldots, \theta_{k}\right)
$$

The goal is to maximize the logarithm of the likelihood function, obtained according to Bayes' theorem:

$$
\log L(\Psi)=\prod_{i=1}^{N} f\left(x_{i} \mid \Psi\right)=\prod_{i=1}^{N} \sum_{k=1}^{K} \frac{\pi_{k}}{\sqrt{2 \pi \sigma_{k}^{2}}} e^{-\frac{\left(x_{i}-\mu_{k}\right)^{2}}{2 \sigma_{k}{ }^{2}}}
$$

Now, let $Z_{i}$ be a random variable, which takes the values $k=1,2, \ldots, K$ with probabilities $\pi_{1}, \pi_{2}, \ldots, \pi_{k}$ respectively. $Z_{i}$ can be considered as the label that indicates the class to which pixel $i$ belongs to. This situation can be represented in an alternate way by defining the random vector $\mathbf{Z}_{\mathbf{i}}=\left(Z_{1}^{(i)}, Z_{2}^{(i)}, \ldots Z_{K}^{(i)}\right)$, where $Z_{k}^{(i)}$ is defined as: 


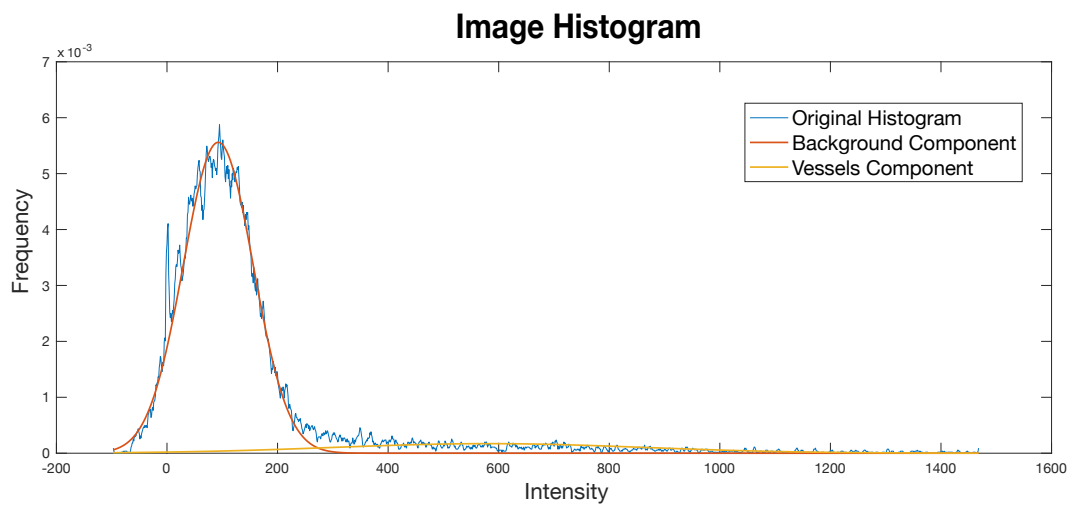

Figure 2. Histogram of a MIP image extracted at the cortical EP of an electrode, considering the portion of volume contained in the first centimeter of the trajectory. It can be noticed that there is a major background component at low intensity values, while higher values represents vessels. The two components have been enhanced by their respective fitted Gaussian distribution.

$$
Z_{k}^{(i)}= \begin{cases}1 & \text { if } X_{i} \in k \\ 0 & \text { otherwise }\end{cases}
$$

With this definition, the $\mathbf{Z}_{\mathbf{i}}$ 's follow a multinomial distribution and using this fact and the Bayes' rule the posterior probability can be obtained as:

$$
\begin{aligned}
P\left(Z_{i}=k \mid x_{i}, \boldsymbol{\Psi}\right) & =\frac{P\left(Z_{i}=k\right) P\left(x_{i} \mid Z_{i}=k, \boldsymbol{\Psi}\right)}{F\left(x_{i}, \mathbf{\Psi}\right)} \\
& =\frac{\pi_{k} f_{k}\left(x_{i} \mid \theta_{k}\right)}{\sum_{m=1}^{K} \pi_{m} f_{m}\left(x_{i} \mid \theta_{m}\right)}=p_{i k}
\end{aligned}
$$

The $p_{i k}$ are called pixel membership because they represent the probability that pixel $i^{\text {th }}$ belongs to the $k^{t h}$ class. To estimate the parameters of each Gaussian class, the Expectation Maximization (EM) approach can be used in order to maximize the likelihood function. In Expectation (E) step, the likelihood function is calculated using the current parameters, while in the Maximization (M) step the parameters are updated. More in detail, the following equations are used for parameters updating:

$$
\begin{gathered}
\pi_{k}^{n+1}=\frac{1}{N} \sum_{i=1}^{N} p_{i k}^{n} \\
\mu_{k}^{n+1}=\frac{1}{N \pi_{k}^{n+1}} \sum_{i=1}^{N} p_{i k}^{n} x_{i} \\
\left(\sigma_{k}^{2}\right)^{n+1}=\frac{1}{N \pi_{k}^{n+1}} \sum_{i=1}^{N} p_{i k}^{n}\left(x_{i}-\mu_{k}^{n+1}\right)^{2}
\end{gathered}
$$

Posterior probabilities $p_{i k}$ have the following expression:

$$
p_{i k}^{n}=\frac{\frac{\pi_{k}^{n} e^{-\frac{\left(x_{i}-\mu_{k}^{n}\right)^{2}}{2\left(\sigma_{k}^{2}\right)^{n}}}}{\sqrt{2 \pi\left(\sigma_{k}^{2}\right)^{n}}}}{\sum_{m=1}^{K} \frac{\pi_{m}^{n} e^{-\frac{\left(x_{i}-\mu_{m}^{n}\right)^{2}}{2\left(\sigma_{m}^{2}\right)^{n}}}}{\sqrt{2 \pi\left(\sigma_{m}^{2}\right)^{n}}}}
$$


The stopping rule, necessary because of the iterative nature of the algorithm, consists in the evaluation of the increment of the logarithm of the likelihood between two following iteration. If it is smaller than a threshold $\epsilon$, then the algorithm stops:

$$
\Delta(\log L(\boldsymbol{\Psi}))=\left|\log \Psi^{n+1}-\log \Psi^{n}\right|<\epsilon
$$

The $X_{i}$ 's independence assumption is relaxed including Markov Random Field in the EM process. $P\left(Z_{i}=k\right)$ can be so defined as:

$$
P\left(Z_{i}=k\right)=\frac{e^{\beta \delta_{i}(k)}}{\sum_{k=1}^{K} e^{\beta \delta_{i}(k)}}
$$

where $\beta>0$ is a parameter controlling the influence of the $i^{t h}$ pixel neighborhood $R_{i}$ and $\delta_{i}(k)$ is the number of neighbors of the $i^{t h}$ pixel that belong to class $k$.

Vessel segmentation is performed on a reduced portion of the image, which is automatically defined around the EP. An example histogram is shown in Fig. 2, where it is possible to notice the presence of a high narrow component at low intensities, representing the background, and a low wide component at high intensities, relative to the vessels. Hence, a two classes GMM-MRF is used, with 8-connected neighborhood, $\beta=1.5$ and $\epsilon=0.1$.

\subsection{Dataset}

In this study, a retrospective analysis on an anonymized data-set of 4 patients is performed. The dataset includes a 3D Cone-Beam Computed Tomography (CBCT) Digital Subtraction Angiography (DSA) ${ }^{1}$ acquired with Oarm 1000 System (Medtronic; Minneapolis, Minnesota, USA) and EP-TP list of electrodes. A total number of 51 images are analyzed, since 51 electrodes were implanted.

\subsection{Validation}

To validate GMM-MRF algorithm, we compared the results against manual segmentation performed under the supervision of an expert surgeon, which served as Gold Standard (GS). We computed indexes relative to Sensitivity $(S e)$, Specificity $(S p)$, Accuracy $(A c c)$, and Dice Similarity Coefficient $(D S C)$. Sensitivity indicates the proportion of True Positive $(T P)$ which have been correctly identified, normalized over the sum of $T P$ and False Negatives $(F N)$ as shown in Eq. 14.

$$
S e=\frac{T P}{T P+T N}
$$

$S e$ represents the vessel pixels which have been correctly classified as vessels. On the other side, $S p$ indicates the proportion of True Negative $(T N)$, normalized over the sum of $T N$ and False Positive $(F P)$ as in Eq. 15. Then, $S p$ indicates how well the background has been classified.

$$
S p=\frac{T N}{T N+F P}
$$

Accuracy $(A c c)$ measures the closeness of the segmentation to the GS, with an index representing the proportion of true results as shown in Eq. 16.

$$
A c c=\frac{T P+T N}{T P+T N+F P+F N}
$$

$D S C$ is based on the superposition of spatial regions, and have been widely used for segmentation algorithms validation. Defining $\left|\Omega_{1}\right|$ and $\left|\Omega_{2}\right|$ as the number of segmented pixels in the two spatial regions, the index is computed as shown in equation 17 .

$$
D S C=\frac{2 *\left|\Omega_{1} \cap \Omega_{2}\right|}{\left|\Omega_{1}\right|+\left|\Omega_{2}\right|}
$$

GMM-MRF were compared also with manual global Thresholding (Th) and classical Gaussian Mixture Model (GMM). All segmentation results were always compared with GS. For each MIP image, the Th value was manually chosen to guarantee the best segmentation based on a qualitative analysis. 
Table 1. Performance measure for Th, GMM and GMM-MRF. Sensitivity $(S e)$, Specificity $(S p)$, Accuracy $(A c c)$ and Dice Similarity Coefficient $(D S C)$ median values (and interquartile ranges) are reported.

\begin{tabular}{cccc}
\hline & Th & GMM & GMM-MRF \\
\hline Se & $0.7608(0.1434)$ & $0.9289(0.0508)$ & $0.8060(0.0722)$ \\
Sp & $0.9927(0.0068)$ & $0.9608(0.0346)$ & $0.9904(0.0082)$ \\
Acc & $0.9642(0.0133)$ & $0.9553(0.0273)$ & $0.9668(0.0108)$ \\
DSC & $0.8403(0.0639)$ & $0.8312(0.0674)$ & $0.8545(0.0381)$ \\
\hline
\end{tabular}

\section{Sensitivity}

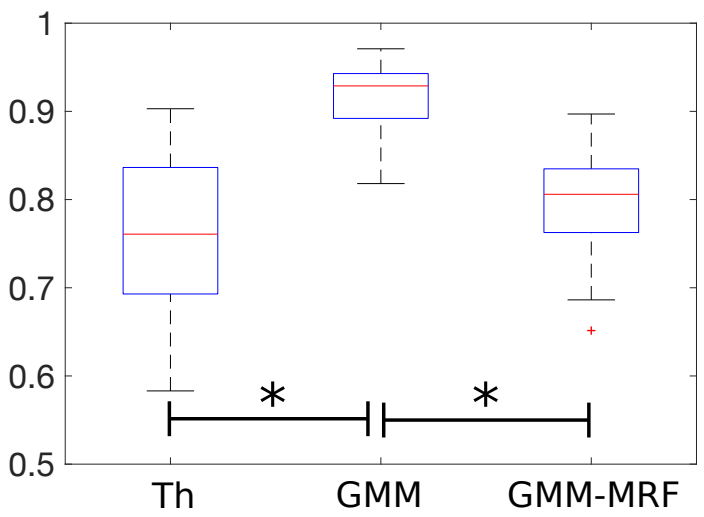

(a)

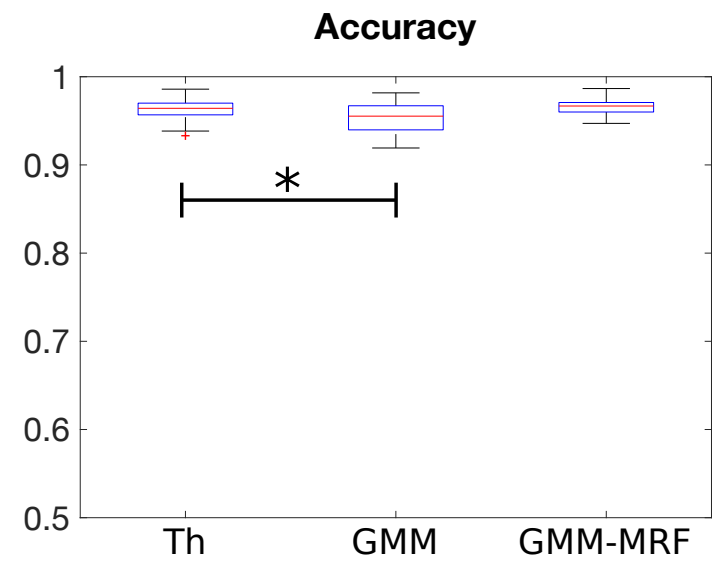

(c)

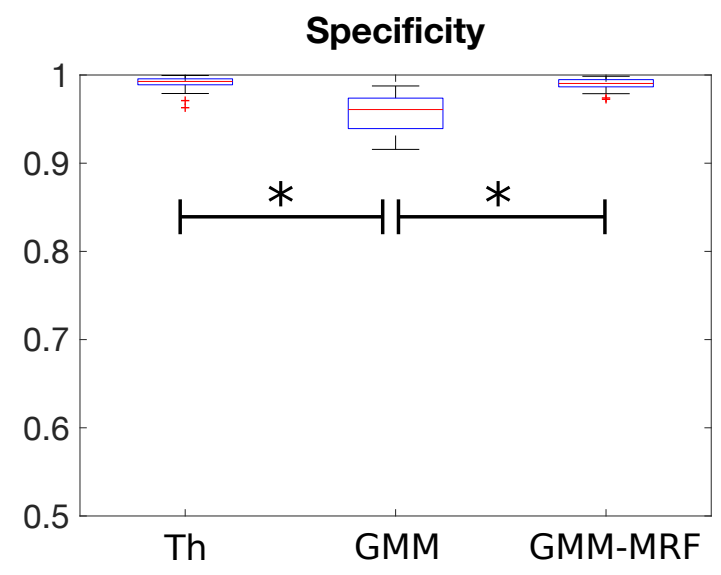

(b)

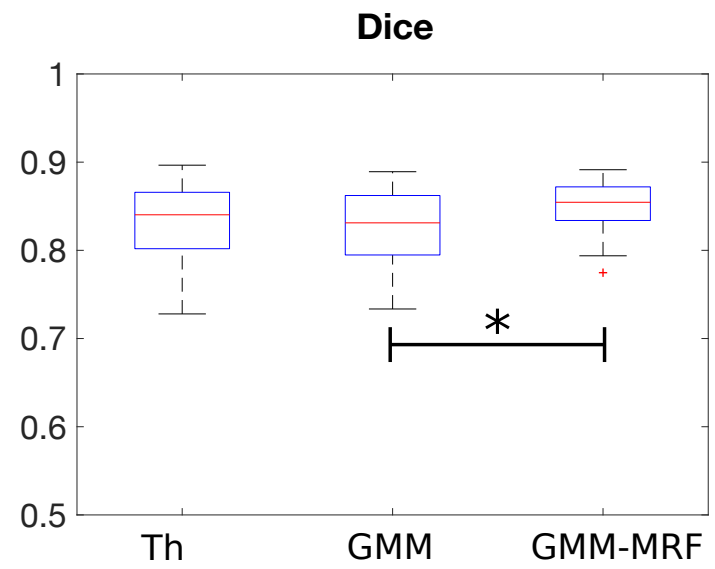

(d)

Figure 3. $S e(3 \mathrm{a}), S p(3 \mathrm{~b}), A c c(3 \mathrm{c})$ and $D S C$ (3d) boxplots for thresholding, GMM and GMM+MRF. Statistical differences are reported.

We applied the Kolmogorov-Smirnov test to check the normality of our data, and consequently a KruskallWallis test and a multiple comparison test ${ }^{17}$ check any statistical difference between the results. Statistical analysis was performed in MATLAB R2016.

\section{RESULTS}

In this section segmentation results in terms of $S e, S p, A c c$ and $D S C$ are presented. $S e, S p, A c c$ and $D S C$ boxplots are shown in Fig. 3. Statistical differences are highlighted. Table 1 shows the $S e, S p, A c c$ and $D S C$ median and inter-quartile range values for Th, GMM and GMM-MRF. Fig. 4 shows the obtained segmentation for a MIP image. 


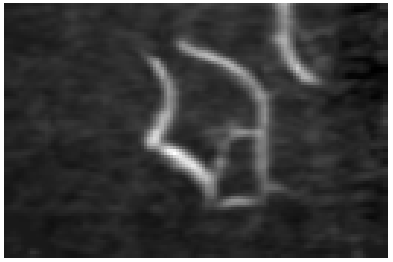

(a)

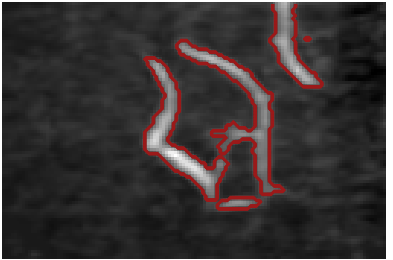

(b)

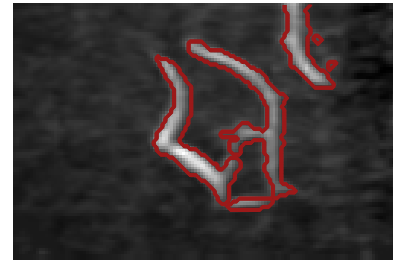

(c)

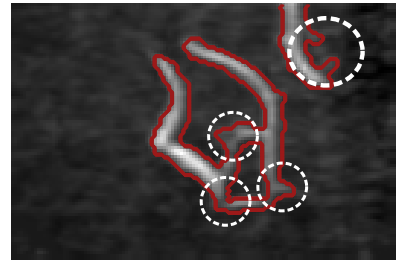

(d)

Figure 4. Segmentation results for a MIP image. (4a) Original MIP image. (4b), (4c), (4d) Segmentation obtained with manual Th, GMM and GMM-MRF, respectively. White dotted circles indicate where the GMM-MRF segmentation was able to reproduce the brain vessel connectivity, overcoming image intensity drop.

\section{DISCUSSION AND FUTURE WORK}

In this work we presented an innovative method to assist surgeons during trajectory planning of electrodes in SEEG. MIP images are used to enhance vessel structures around the electrode, while an automatic segmentation method allows to eliminate possible unconnected noisy pixels.

From the performed analysis, generally GMM-MRF showed better performance with respect to the other methods. Both methods, Th and GMM-MRF were found statistically different with respect the indexes computed, showing better performances in terms of $S p, A c c$ and $D S C$. In addition, by visually inspecting segmentation results, GMM-MRF was able to better represent the connected nature of the brain vessels, especially in presence of image intensity drop, typical of MIP images (e.g. Fig. 4) without oversegment vessels.

Th and GMM-MRF showed similar performances, and no statistical difference were found between the two methods. It is reasonable that Th performed so well due to the high contrast between the background and vessels, guaranteed by contrast medium and MIP. However, selecting an optimal threshold could not be an immediate task and it is affected by inter-subject variability. On the contrary, GMM-MRF led to better results in terms of segmentation, and represents a completely automatic method able to identify vessel structures and compensate noisy pixels introduced by MIP.

Future work will explore the possibility to extend this method for volume segmentation. A similar approach will be applied by generating MIP images iteratively using contiguous portions of the volume along electrode principal axis and merge the results to segment the whole vascular structure. Moreover, the approach here presented can be easily integrated in an automatic trajectory planner, such as the one presented in, ${ }^{4}$ to further automatically support surgeons in the definition of safe electrode trajectories.

\section{REFERENCES}

[1] Cardinale, F., Pero, G., Quilici, L., Piano, M., Colombo, P., Moscato, A., Castana, L., Casaceli, G., Fuschillo, D., Gennari, L., Cenzato, M., Lo Russo, G., and Cossu, M., "Cerebral angiography for multimodal surgical planning in epilepsy surgery: description of a new three-dimensional technique and literature review," World neurosurgery 84(2), 358-367 (2015).

[2] Cardinale, F., Casaceli, G., Raneri, F., Miller, J., and Lo Russo, G., "Implantation of stereoelectroencephalography (seeg) electrodes: a systematic review," Clinical Neurophysiology , 490-502 (2016).

[3] Cardinale, F., Cossu, M., Castana, L., Casaceli, G., Schiariti, M. P., Miserocchi, A., Fuschillo, D., Moscato, A., Caborni, C., Arnulfo, G., et al., "Stereoelectroencephalography: surgical methodology, safety, and stereotactic application accuracy in 500 procedures," Neurosurgery 72(3), 353-366 (2013).

[4] De Momi, E., Caborni, C., Cardinale, F., Casaceli, G., Castana, L., Cossu, M., Mai, R., Gozzo, F., Francione, S., Tassi, L., et al., "Multi-trajectories automatic planner for StereoElectroEncephaloGraphy (SEEG)," International journal of computer assisted radiology and surgery 9(6), 1087-1097 (2014).

[5] Sparks, R., Zombori, G., Rodionov, R., Nowell, M., Vos, S. B., Zuluaga, M. A., Diehl, B., Wehner, T., Miserocchi, A., McEvoy, A. W., et al., "Automated multiple trajectory planning algorithm for the placement of stereo-electroencephalography (SEEG) electrodes in epilepsy treatment," International Journal of Computer Assisted Radiology and Surgery , 1-14 (2016). 
[6] Fraz, M. M., Remagnino, P., Hoppe, A., Uyyanonvara, B., Rudnicka, A. R., Owen, C. G., and Barman, S. A., "Blood vessel segmentation methodologies in retinal images-a survey," Computer methods and programs in biomedicine 108(1), 407-433 (2012).

[7] De Momi, E., Caborni, C., Cardinale, F., Castana, L., Casaceli, G., Cossu, M., Antiga, L., and Ferrigno, G., "Automatic trajectory planner for StereoElectroEncephaloGraphy procedures: a retrospective study," Biomedical Engineering, IEEE Transactions on 60(4), 986-993 (2013).

[8] Du, X., Ding, H., Zhou, W., Zhang, G., and Wang, G., "Cerebrovascular segmentation and planning of depth electrode insertion for epilepsy surgery," International journal of computer assisted radiology and surgery 8(6), 905-916 (2013).

[9] Frangi, A. F., Niessen, W. J., Vincken, K. L., and Viergever, M. A., "Multiscale vessel enhancement filtering," in [International Conference on Medical Image Computing and Computer-Assisted Intervention], 130-137, Springer (1998).

[10] Shamir, R. R., Joskowicz, L., Antiga, L., Foroni, R. I., and Shoshan, Y., "Trajectory planning method for reduced patient risk in image-guided neurosurgery: concept and preliminary results," in [SPIE Medical Imaging], 76250I-76250I, International Society for Optics and Photonics (2010).

[11] Zuluaga, M. A., Rodionov, R., Nowell, M., Achhala, S., Zombori, G., Cardoso, M. J., Miserocchi, A., McEvoy, A. W., Duncan, J. S., and Ourselin, S., "SEEG trajectory planning: Combining stability, structure and scale in vessel extraction," in [International Conference on Medical Image Computing and ComputerAssisted Intervention], 651-658, Springer (2014).

[12] Gonzalez-Martinez, J., Mullin, J., Vadera, S., Bulacio, J., Hughes, G., Jones, S., Enatsu, R., and Najm, I., "Stereotactic placement of depth electrodes in medically intractable epilepsy: Technical note," Journal of neurosurgery 120(3), 639-644 (2014).

[13] Cardinale, F., Miserocchi, A., Moscato, A., Cossu, M., Castana, L., Schiariti, M., Gozzo, F., Pero, G., Quilici, L., Citterio, A., et al., "Talairach methodology in the multimodal imaging and robotics era," Stereotaxy and Epilepsy Surgery. John Libbey Eurotext, 245-272 (2012).

[14] Fedorov, A., Beichel, R., Kalpathy-Cramer, J., Finet, J., Fillion-Robin, J.-C., Pujol, S., Bauer, C., Jennings, D., Fennessy, F., Sonka, M., et al., "3D Slicer as an image computing platform for the Quantitative Imaging Network," Magnetic resonance imaging 30(9), 1323-1341 (2012).

[15] Rue, H. and Held, L., [Gaussian Markov random fields: theory and applications], CRC Press (2005).

[16] Anderson, C. M., Saloner, D., Tsuruda, J. S., Shapeero, L. G., and Lee, R. E., "Artifacts in maximumintensity-projection display of MR angiograms.," AJR. American journal of roentgenology 154(3), 623-629 (1990).

[17] Hochberg, Y. and Tamhane, A. C., "Multiple comparison procedures," (2009). 